\title{
A Xylophone Bar Magnetometer for micro/pico satellites
}

\author{
Hervé Lamy*, Véronique Rochus ${ }^{\dagger}$, Innocent Niyonzima ${ }^{\dagger}$ and Pierre Rochus ${ }^{\ddagger}$ \\ *Belgian Institute for Space Aeronomy \\ Avenue Circulaire 3, 1180 Brussels, Belgium \\ ${ }^{\dagger}$ Department of Aerospace and Mechanical Engineering, \\ University of Liège, Chemin des Chevreuils 1 (B52/3), 4000 Liège, Belgium \\ ${ }^{\ddagger}$ Centre Spatial de Liège, \\ University of Liège, 4031 Angleur, Belgium
}

\begin{abstract}
The Belgian Institute of Space Aeronomy (BIRAIASB), "Centre Spatial de Liège" (CSL), "Laboratoire de Techniques Aéronautiques et Spatiales" (LTAS) of University of Liège, and the Microwave Laboratory of University of Louvain-LaNeuve (UCL) are collaborating in order to develop a miniature version of a xylophone bar magnetometer (XBM) using Microelectromechanical Systems (MEMS) technology. The device is based on a classical resonating xylophone bar. A sinusoidal current is supplied to the bar oscillating at the fundamental transverse resonant mode of the bar. When an external magnetic field is present, the resulting Lorentz force causes the bar to vibrate at its fundamental frequency with an amplitude directly proportional to the vertical component of the ambient magnetic field. In this paper we illustrate the working principles of the $\mathrm{XBM}$ and the challenges to reach the required sensitivity in space applications (measuring magnetic fields with an accuracy of approximately of $0.1 \mathrm{nT}$ ). The optimal dimensions of the MEMS XBM are discussed as well as the constraints on the current flowing through the bar. Analytical calculations as well as simulations with finite element methods have been used. Prototypes have been built in the Microwave Laboratory using Silicon on Insulator (SOI) and bulk micromachining processes. Several methods to accurately measure the displacement of the bar are proposed.
\end{abstract}

\section{INTRODUCTION}

Magnetic fields play a key role in many aspects of the solar-terrestrial interactions. For example, during geomagnetic activity, charged particles precipitate along geomagnetic field lines and produce spectacular aurora. Strong sheets of fieldaligned currents (FACs) associated with these precipitations produce local perturbations of the geomagnetic field. A magnetometer onboard a spacecraft crossed by these current sheets will record the magnetic field perturbations and will provide a measure of the field aligned current using the Maxwell's equation $\vec{\nabla} \wedge \vec{B}=\vec{J}$. With a single spacecraft stationary current sheet may be obtained. However this situation is not always observed. In some cases, spatial and temporal variations of the magnetic field cannot be discriminated. Currently the separation of satellites in multi-spacecraft missions like Cluster or Themis is usually larger than the width of the current sheet. This example illustrates the importance of multipoint measurements with a fleet of micro- or pico-spacecraft with small separations.
Since launch costs represent a significant fraction of the total mission expenditures, reducing such costs necessitates careful consideration of instrument and spacecraft component miniaturization. The goal of this research is to build a sensitive, low mass, low size and low consumption magnetometer to embark onboard a fleet of micro-, nano- or pico-satellites in order to carry out these multi-point measurements.

Three main types of magnetometers have been traditionally used in space missions: the fluxgate magnetometer (FGM), the search-coil magnetometer (SCM) and the vector helium magnetometer (VHM). Efforts to miniaturize size and mass of these magnetic sensors have been limited by fabrication difficulties and loss of sensitivity. Moreover, they are always located at the end of long booms to avoid magnetic perturbations from the spacecraft itself, a configuration that is hardly compatible with nano- or pico-satellites.

In the framework of the Solar Terrestrial Center of Excellence (STCE), the Belgian Institute for Space Aeronomy (BISA) is studying the feasibility of developing and constructing a magnetometer for space applications suited to miniaturization. With "Centre Spatial de Liège (CSL)", "Laboratoire de Techniques Aéronautiques et Spatiales" (LTAS) of University of Liège, and Microwave Laboratory of University of LouvainLa-Neuve (UCL), the objective is to develop a Xylophone Bar Magnetometer (XBM) in which the displacement of a beam is directly proportional to the magnitude of one component of the ambient magnetic field. The specific design of this magnetometer makes it particularly suitable to be fabricated at the size of Microelectromechanical Systems (MEMS). This study benefits from previous works initiated at the Applied Physics Laboratory of the John Hopkins University (Givens et al 1996, Wickenden et al 1997, Zanetti et al 1998, Oursler et al 1999, Wickenden et al 2003).

The challenging task with this $\mathrm{XBM}$ is to reach the subnanotesla accuracy and qualify it for space applications. Another goal we would like to achieve is to use a set of such miniature magnetometers in a clever way to accurately determine the electromagnetic contribution of the electronics onboard a spacecraft. On one hand, this would reduce the magnetic cleanliness requirements currently imposed on spacecraft instruments and on the other hand, this would prevent the use 


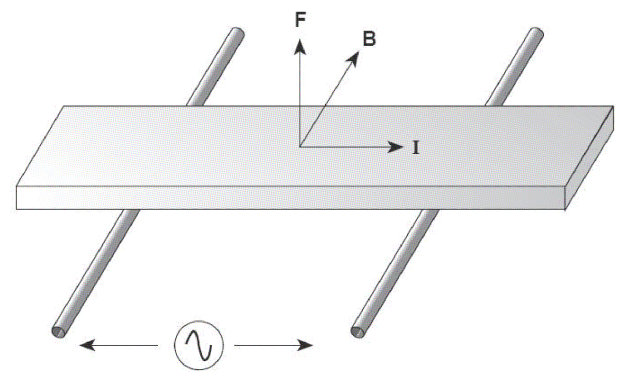

Fig. 1. Operating principle of a Xylophone Bar Magnetometer

of large booms for magnetometers.

In section II, the working principles of the XBM are described. An analytic study of the parameters influencing the deflection of the bar is discussed in section III whilst section IV is devoted to simulations with finite element methods (FEM). In section V, we describe a first XBM MEMS prototype manufactured at the Microwave Laboratory as well as preliminary measurements of the curvature of the structure. Several methods to accurately measure the displacement of the bar are considered in section VI. We conclude with some perspectives about additional modeling with simulations.

\section{WORKING PRINCIPLES OF THE XBM}

The XBM magnetometer is based on a classical resonating xylophone bar. This relatively simple device uses the Lorentz force to measure one component of the ambient magnetic field. It consists of a thin conductive xylophone bar supported at the nodes of its fundamental mode of mechanical vibration by two arms bonded to the bar to provide low-resistance electrical contacts (see Figure 1). The nodes are located at $22.4 \%$ of the bar's length from each unclamped end.

A sinusoidal current is supplied to the bar through these arms, oscillating at the fundamental transverse resonant mode of the bar $f_{0}$ :

$$
f_{0}=\frac{22.4}{2 \pi} \sqrt{\frac{E I_{a}}{\omega L^{4}}}=\frac{1.029 b}{L^{2}} \sqrt{\frac{E}{\rho}}
$$

where $\mathrm{E}$ is the Young's modulus $\left(N / \mathrm{m}^{2}\right), L$ the bar length, $I_{a}$ the area moment of inertia $\left(=a b^{3} / 12\right.$ for a rectangular beam), $\omega$ the mass per unit length $(\rho=\omega a b)$, and $\rho, \mathrm{a}, \mathrm{b}$ are the mass density, width and thickness of the bar, respectively. When a current $I$ is driven through the xylophone bar in the presence of an external magnetic field $B_{\text {ext }}$, a resulting Lorentz force applied to the bar makes it vibrate vertically.

$$
\vec{F}=I \overrightarrow{L_{s}} \wedge \overrightarrow{B_{\text {ext }}}
$$

where $\overrightarrow{L_{s}}$ is a vector whose magnitude $L_{s}$ is the length of the xylophone bar between the supports $\left(L_{s} \sim 0.552 L\right)$. When the frequency of the current is set at the fundamental transverse resonant frequency of the bar, the deflection of the bar is strongly enhanced and its amplitude in the middle of the bar is given by

$$
d\left(f_{0}\right)=\frac{5 F L_{s}^{4}}{384 E I_{a}} Q=1.206 \times 10^{-3} \times \frac{F L^{4}}{E I_{a}} Q
$$

where $Q$ is the mechanical quality factor which is determined by different parameters depending on bar material, manufacturing process, but also includes various types of dampings such as air damping, support damping, thermoelastic damping and surface damping. Equation (3) is obtained by modeling the bar as a mass-spring-dashpot system submitted to a static deformation. Another approach to obtain the theoretical displacement of the bar is to solve the Euler-Bernouilli equation of a free-free bar submitted to a dynamic deformation (Niyonzima 2009). In this case, the displacement of the bar is given by

$$
d\left(f_{0}\right)=1.747 \times 10^{-3} \times \frac{F L^{4}}{E I_{a}} Q
$$

The ratio between equations (4) and (3) is equal to 1.446 . Both theoretical values will be compared to values obtained with simulations in section IV.

From equations (2), (3) and (4), it can be seen that the amplitude of the deflection of the bar is linearly proportional to the magnetic field component $B$ parallel to the surface of the xylophone bar and normal to the direction of the drive current. This device is therefore intrinsically linear unlike many other magnetometers. In principle, it also has a very wide dynamical range: magnetic field intensities from nanoteslas to teslas could be measured by simply adjusting the current amplitude. However, the maximum intensity of the current that can flow through the bar depends on the importance of the Joule effect and of the thermomechanical coupling. Indeed, a too large elevation of temperature inside the bar could result in significant modifications of its mechanical properties. This thermomechanical coupling is briefly discussed in section IV devoted to simulations. The influence of the other parameters of the bar (dimensions, Young modulus, quality factor) on its deflection are discussed in section III.

Because the other vibration modes of the bar have a very different frequency and are not excited at this frequency $f_{0}$, this technique discriminates against these components of the magnetic field extremely well so that any second-order cross coupling between different field components is extremely small. A three-axis sensor can be constructed with three xylophone bars operating at different frequencies.

The variation of the amplitude of the bar deflection as a function of the frequency of the driving current in a constant external magnetic field $B$ is given by

$$
d=d\left(f_{0}\right) \times \frac{1}{\sqrt{\left[1-\left(\frac{f}{f_{0}}\right)^{2}\right]^{2}+\left(\frac{f}{Q f_{0}}\right)^{2}}}
$$

As the frequency is chosen as the fundamental mechanical resonance frequency, the displacement amplitude is enhanced, 
reaching a maximum value given by $d\left(f_{0}\right)$, while the phase angle displays a $180^{\circ}$ shift.

The amplitude of the deflection of the xylophone bar can be measured by established detection techniques such as optical and capacitive methods or by new techniques using plasmons or magnetostrictive material. They will be shortly discussed in section VI.

\section{PARAMETRIC STUDY OF THE DEFLECTION}

In this section we discuss different parameters influencing the deflection $d$, namely, the electric current $I$, the Young modulus $E$, the quality factor $Q$ and the dimensions $L, a$ and $b$ of the bar.

\section{A. Influence of the current: Joule effect and thermoelectric coupling}

According to equation (3) or (4), the larger the current flowing through the bar, the larger its deflection. However, the maximum value of the electrical current flowing into the bar is in practice limited by the Joule effect and the thermomecanical coupling. The increase of temperature resulting from the Joule effect can on one hand modify the thermal and electrical conductivites of the material (respectively $\kappa$ and $\sigma$ ), and on another hand the mechanical properties of the bar via the thermal coefficient of expansion $\alpha$ (which can modify the dimensions of the structure, therefore its eigenfrequencies) or via the Young's modulus. These couplings can better be accounted for with FEM simulations. In section IV, a simpler method will be used with a mechanical calculation carried out after a thermo-electromagnetic study.

However, as a first step, we make a simple estimation of the maximal value of the current $I_{\max }$ by using an electric circuit analogy of the system insulator+bar+arms. In this analogy, conduction heat transfer is modeled with thermal resistances $R_{x}=\frac{w_{x}}{\kappa_{x} S_{x}}$ where $x$ stands either for the insulator, the arm or the bar. $w$ is the width, $\kappa$ the thermal conductivity and $S$ the cross section. A discrete distribution of temperature is assumed in several nodes (see Fig 2). The temperature profile is determined by solving a set of Kirchoff laws at the nodes when a voltage is applied through the arms. This temperature profile is then used to update the values of electric and thermal conductivitites. Electric resistivity was represented by an empirical linear relationship $\rho=\rho_{0} \times\left(1+\alpha\left(T-T_{0}\right)\right)$ where $\rho_{0}$ is the resistivity at the room temperature $T_{0}\left(\rho_{0} \sim 2,28 \times 10^{-8} \Omega \mathrm{m}\right.$ and $\alpha \sim 0.004 \mathrm{~K}^{-1}$ in the case of Aluminium). The thermal conductivity $\kappa$ was then calculated with the Wiedemann-Franz law

$$
\frac{\kappa}{\sigma T}=\frac{\pi^{2} k_{b}^{2}}{3 e^{2}}
$$

where $k_{b}$ and $e$ are respectively the Boltzmann constant and the charge of the electron. This empirical law is valid in metals where the heat conduction is mainly ensured by free electrons. It would not be valid in semi-conductors like silicon where heat conduction is also due to phonons. Also, experiments have shown that it is valid at high and low

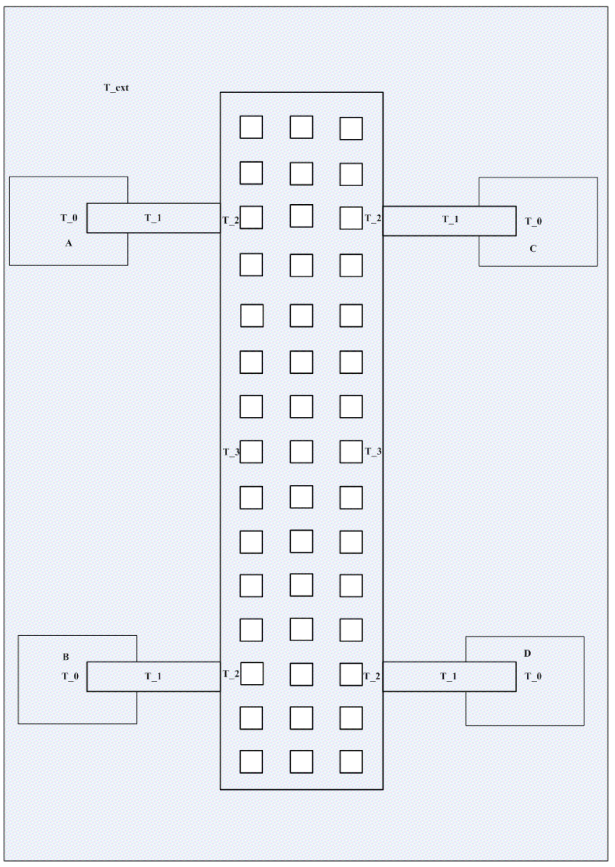

Fig. 2. Sketch of the electric circuit analogy used to estimate the maximal value of the current that can flow into the bar. The actual bar is filled with holes to facilitate the etching of the insulator but these holes have not been modeled in the electric circuit.

temperatures but that there are slight deviations from this law at intermediate temperatures. Despite these limitations, this approach is good enough for a first-order estimation. Once we have the discrete temperature distribution in our full system, we modify the electrical and thermal conductivities and solve the Kirchoff laws again until we reach a steady-state. In this simple approach, the resolution of the temperature distribution is too crude but we take into account in a rather simple way the coupling between thermal and electrical fields. Finally we assume that heat transport is mainly due to conduction and that heat losses by convection and radiation are negligible. This assumption is correct because of the very small size of the emitting surface and if we work in a rarefied medium.

These calculations with the electric circuit analogy were carried out on the system sketched in Figure 2. Dimensions of the bar are $502 \mu \mathrm{m} \times 54 \mu \mathrm{m} \times 2.5 \mu \mathrm{m}$ and those of the arms are $15 \mu \mathrm{m} \times 4 \mu \mathrm{m} \times 2.5 \mu \mathrm{m}$. Thermal and electrical conductivities for the Aluminium at room temperature are $237 \mathrm{Wm}^{-1} \mathrm{~K}^{-1}$ and $37.7 \times 10^{6} \mathrm{~S} \mathrm{~m}^{-1}$. Thermal conductivity for the insulator $\mathrm{SiO}_{2}$ is $1.38 \mathrm{~W} \mathrm{~K}^{-1}$. Figure 3 shows the maximum temperature in the bar $\left(T_{3}\right.$ in Figure 2 located at half the distance between the nodes) as a function of the voltage applied between the nodes. Two curves are shown according to whether some thermo-electrical coupling is taken into account or not. From these curves we see that an increase of temperature of $10 \mathrm{~K}$ is obtained for an applied voltage of approximately $0.015 \mathrm{~V}$ which corresponds to a current of $\sim 0.15 \mathrm{~A}$ for an aluminium bar with the aforementionned dimensions. 


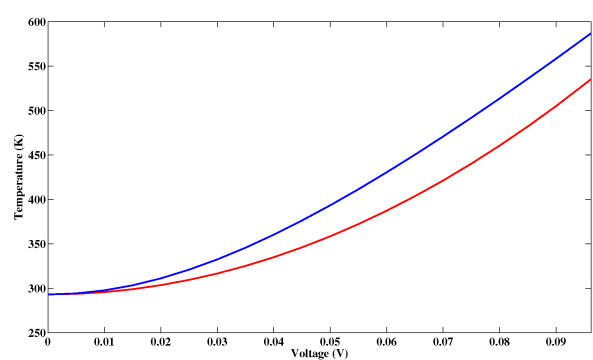

Fig. 3. Maximum temperature in the middle of the bar as a function of the RMS voltage applied between the nodes. We consider an aluminium bar of dimensions $502 \mu \mathrm{m} \times 54 \mu \mathrm{m} \times 2.5 \mu \mathrm{m}$ with $15 \mu \mathrm{m} \times 4 \mu \mathrm{m} \times 2.5 \mu \mathrm{m}$ supporting arms. The red curve is obtained when the Wiedemann-Franz law is not taken into account (no thermo-electrical coupling) while the blue curve is obtained when this law is used.

\section{B. Influence of the Young Modulus}

The lower the Young's modulus $E$, the lower the first mechanical resonance frequency of the bar and the larger its deflection. At first sight, we should therefore choose a material with a small value of $E$. However, in addition, we must also select a material with large thermal and electrical conductivities and which can actually be used in MEMS micromachining.

Our choice to realize the first prototype in the Microwave Laboratory was to use Aluminium which has a relatively small Young's modulus $(E=70 \mathrm{GPa})$ and is a very good heat and electrical conductor. However, as we will see below, the bulk micromachining used at the Microwave Laboratory introduced a lot of residual stresses in the bar due to the large difference of the thermal expansion coefficient of the materials used for the bar and for the substrate $\left(\alpha=23 \times 10^{-6} K^{-1}\right.$ for aluminium and $\alpha=3 \times 10^{-6} K^{-1}$ for silicon).

\section{Influence of the quality factor $Q$}

In this section, we follow an approach similar to the work of Naeli \& Brand (2009). Four sources of dampings are considered : air damping, support damping, thermoelastic damping and surface damping.

Air damping is due to the loss of energy resulting from the interaction of the bar with the surrounding air. At low air pressure, in the molecular regime, the damping is due to collisions with individual air molecules. The quality factor

$$
Q_{\text {air }}=\frac{\mu_{n}^{2}}{k_{m} P}\left(\frac{b}{L}\right)^{2} \sqrt{\frac{E \rho}{12}}
$$

varies with the air pressure $P$ (Blom et al, 1992). $\mu_{n}$ is a constant equal to 4.73 for the first bending mode of a free-free bar and $k_{n}=\sqrt{\frac{32 M}{9 \pi R T}}$ where $R$ is the gas constant, $M$ the molecular mass of the air and $T$ the temperature (in $\mathrm{K}$ ). At higher pressure, in the viscous regime, the air acts as a viscous fluid that brakes the displacement of the bar. The bar is then modeled as a string of resonant spheres for which the quality factor does not depend anymore on $P$ (Hosaka et al 1995, Naeli \& Brand 2009). Of course, in space, the contribution of air damping will be negligible but for other potential groundbased applications, we keep it as a possible loss source.

Support damping is usually one of the most important dissipation mechanism in MEMS. It represents the mechanical energy dissipated via the coupling to the support structure. In the case of our XBM, the supporting arms are connected at the nodes of the first mode shape of the bar and in theory the damping is equal to zero. Due to the finite width of the supporting arms, this assumption is not completely correct. However, due to the very small width of the supporting arms ( $\sim 4 \mu \mathrm{m}$ in our prototype) compared to the length of the bar $(\sim 500 \mu \mathrm{m})$, we can safely neglect the contribution of support damping.

Thermoelastic damping (TDE) is the loss of energy caused by the coupling between the thermal and the strain fields. When the bar is submitted to bending, the two opposite sides undergo opposite deformations, the upper and lower sides being alternatively compressed or stretched. A gradient of temperature is generated accross the thickness of the bar and a relaxation mechanism can occur. TDE is important when the vibration frequency is of the same order as the thermal relaxation rate, otherwise the vibrations can be considered either as isothermal or adiabatic. For the modelisation of TDE, the model of Lifshitz \& Roukes (2000) is used such that the quality factor is predicted by

$$
Q_{\mathrm{TED}}^{-1}=\frac{E \alpha^{2} T_{0}}{c_{v}}\left[\frac{6}{\zeta^{2}}-\frac{6}{\zeta^{3}} \frac{\sinh (\zeta)+\sin (\zeta)}{\cosh (\zeta)+\cos (\zeta)}\right]
$$

where $\zeta=b \sqrt{\frac{\omega_{0, n}}{2 \chi}}, T_{0}$ the equilibrium temperature and $c_{v}$ the heat capacity at constant volume. $\omega_{0, n}=\mu_{n}^{2} \sqrt{\frac{E I_{a}}{m L^{4}}}$ are the isothermal eigenfrequencies of the bar and $\chi=\frac{\kappa}{c_{v}}$. An order of magnitude of the thermal relaxation time $\tau$ can be obtained by assuming that the variation of temperature is mainly due to conduction. In this case, we have :

$$
\tau \sim \frac{\rho c_{v} b^{2}}{\kappa}
$$

For the case of aluminium $\left(\rho=2.71 \times 10^{3} \mathrm{Kg} \mathrm{m}^{-3}, c_{v}=\right.$ $900 \mathrm{~J} \mathrm{Kg}^{-1} \mathrm{~K}^{-1}$ and $\kappa=237 \mathrm{~W} \mathrm{~m}^{-1} \mathrm{~K}^{-1}$ ), the inverse of the thermal relaxation time $1 / \tau$ varies between $50 \mathrm{MHz}$ and 120 $\mathrm{KHz}$ for bar with thicknesses between 1 and $20 \mu \mathrm{m}$. According to this model, we conclude that the thermoelastic damping is negligible for a bar with dimensions of $502 \mu \mathrm{m} \times 54 \mu \mathrm{m} \times$ $2.5 \mu \mathrm{m}$ because the first eigenfrequency of such a bar is on the order of $100 \mathrm{KHz}$. In this case, heat is transferred between the sides of the bar much quicker than the time needed for an oscillation. The bar is isothermal.

Surface damping might become the predominant type of damping when the thickness of the structure becomes very small, resulting in a large surface to volume ratio. In this case, energy losses are due to surface effects such as adsorption. Yang et al (2002) model the inverse of the quality factor by

$$
Q_{\text {surf }}^{-1}=2 \delta \frac{E_{2}^{s}}{E} \frac{3 a+b}{a b}
$$




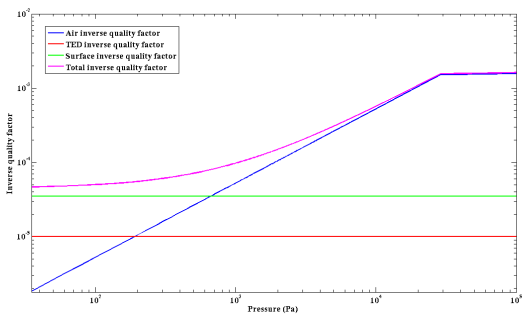

Fig. 4. Inverse quality factors as a function of pressure for air damping, thermoelastic damping and surface damping. Calculations have been carried out for a $502 \mu \mathrm{m} \times 54 \mu \mathrm{m} \times 2.5 \mu \mathrm{m}$ bar with $15 \mu \mathrm{m} \times 4 \mu \mathrm{m} \times 2.5 \mu \mathrm{m}$ supporting arms. Temperature is $293 \mathrm{~K}$.

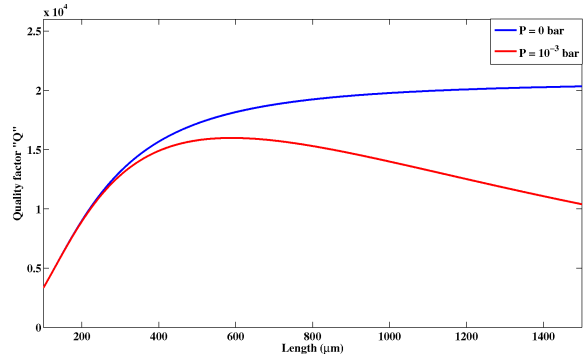

Fig. 5. Quality factor of an aluminium bar as a function of the length. Width $a=54 \mu \mathrm{m}$ and thickness $b=2.5 \mu \mathrm{m}$. Dimensions of supporting arms are $15 \mu \mathrm{m} \times 4 \mu \mathrm{m} \times 2.5 \mu \mathrm{m}$. Temperature is $293 \mathrm{~K}$.

where $E_{2}^{s}$ is a property of the adsorbate layer. Some experiments on $20 \mu \mathrm{m}$ thick beams have shown that the product $\delta E_{2}^{s}$ is close to 1 , a value that we will assume in the following. The exact value of this parameter has to be determined experimentally.

Taking into account the various dampings, the total quality factor is calculated as

$$
Q_{\mathrm{tot}}^{-1}=Q_{\mathrm{air}}^{-1}+Q_{\mathrm{TED}}^{-1}+Q_{\mathrm{surf}}^{-1}
$$

where we have already neglected the support damping. Figure 4 illustrates the inverse of the quality factor as a function of the pressure for the three damping mechanisms. At low pressure, air damping becomes negligible. The major parameter that will then determine whether TED or surface damping dominates is the thickness of the bar. For our $\mathrm{XBM}$, the thickness is small and so the surface damping will be predominant. Since this mechanism is poorly described analytically, the actual quality factor will have to be carefully measured experimentally.

\section{Influence of the dimensions of the bar}

In equation (3), the deflection of the bar is a function of its dimensions via $L$ (or $L_{s}$ ), the area moment of inertia $I_{a}$ and the quality factor $Q$. Figures 5, 6 and 7 respectively illustrate the variation of the quality factor with the length $L$, the width $a$ and the thickness $b$ of the bar. Two cases are shown : no air pressure and air pressure of $10^{-3}$ bar $\left(=10^{3} \mathrm{~Pa}\right)$.

The displacement of the bar increases strongly when the length of the bar increases due to the $L^{4}$ dependance. For

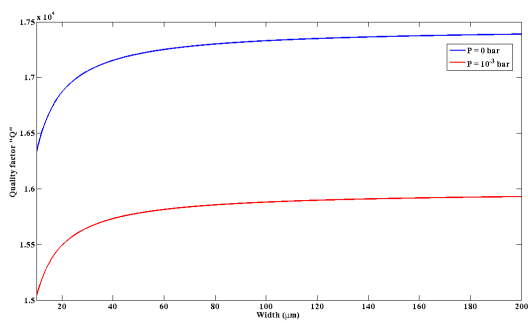

Fig. 6. Quality factor of an aluminium bar as a function of the width. Length $L=502 \mu \mathrm{m}$ and thickness $b=2.5 \mu \mathrm{m}$. Dimensions of supporting arms are $15 \mu \mathrm{m} \times 4 \mu \mathrm{m} \times 2.5 \mu \mathrm{m}$. Temperature is $293 \mathrm{~K}$.

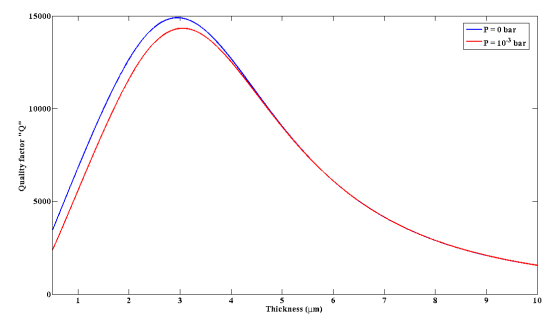

Fig. 7. Quality factor of an aluminium bar as a function of the thickness Length $L=502 \mu \mathrm{m}$ and width $a=54 \mu \mathrm{m}$. Dimensions of supporting arms are $15 \mu \mathrm{m} \times 4 \mu \mathrm{m} \times 2.5 \mu \mathrm{m}$. Temperature is $293 \mathrm{~K}$.

non-zero pressure, there is an optimal value of the length ( $L \sim 500 \mu \mathrm{m}$ giving the highest possible quality factor (see Figure 5). Of course the length of the bar cannot be larger than a maximum value above which the bar will stick to the substrate. The width of the bar has relatively few importance both on the quality factor (see Figure 6) and on the displacement of the bar (because the dependance of $I_{a}$ with $a$ is only linear) but it must be at least of the order of $50 \mu \mathrm{m}$ to have the largest $Q$ value. The simulations presented in section IV will also indicate that the width is important for the correct calculation of the bar eigenfrequencies. Finally, there is also an optimal value of the thickness $(b \sim 2.5 \mu \mathrm{m})$ which maximizes the quality factor (see Figure 7). The thickness also has a strong impact on the displacement of the bar because of the $b^{3}$ dependance of the $I_{a}$ factor. Thinner bars produce larger values of the bar displacement.

Therefore, in the simulations presented below, the length of the bar $L$ will always be of the order of $500 \mu \mathrm{m}$, the width of the bar $a$ of the order of $50 \mu \mathrm{m}$ and the thickness of the bar $b$ lower or equal to $2.5 \mu \mathrm{m}$.

\section{Simulations With Finite ElEMENT METhods}

These FEM simulations have been carried out with Oofelie driven by Samcef. Oofelie is a finite element multi-physics software which allows to model the couplings between various fields. In the XBM, the sinusoidal electric current flowing through the bar induces a temperature increase via a Joule effect, which also modifies the thermal and electrical conductivities of the bar and therefore changes the current distribution inside the bar. There is a thermo-electromagnetic coupling. 
The temperature increase inside the bar can also modify its mechanical properties such as its dimensions and its Young modulus, and therefore its eigenfrequencies. We adopted the following procedure: first, a thermo-electromagnetic steadystate equilibrium is computed. Then, the eigenfrequencies of the bar are calculated by assuming small perturbations of this steady state and carrying out a linear analysis around the equilibrium solution.

Due to the symmetry of the XBM, all these simulations were done only on a quarter of the whole structure (one half in length and one half in width) to save computational time.

\section{A. Influence of the holes in the bar}

As will be described in section V, the bar in our MEMS $\mathrm{XBM}$ prototype is designed with many holes (see also Figure 2 for a sketch) in order for the etchant to easily access the sacrificial $\mathrm{SiO}_{2}$ layer and release the structure during the micromachining process. The dimensions of these holes are $2 \mu \mathrm{m} \times 2 \mu \mathrm{m}$ and their separation is $5 \mu \mathrm{m}$. So, before running the simulations described above, a mechanical simulation is carried out to check their influence on the calculations of the eigenfrequencies. Furthermore, since the presence of these holes strongly increase the computational time (because the size of the mesh must be smaller than the size of a hole), it is highly desirable to make simulations with a full structure (with no holes) instead of the more complicated structure with holes. For that purpose a comparison is made between the values of the first eigenfrequency obtained with two mechanical simulations : the first one is obtained for the structure with holes using a finer mesh size while the second is obtained for the full structure with no holes but with electrical, mechanical and thermal properties modified as follows :

$$
x=x_{0} \times \frac{V_{h}}{V_{0}}
$$

where $V_{h}$ is the volume of the bar with holes and $V_{0}$ is the volume of the bar without holes. $x$ stands for the electrical conductivity $\sigma$, the thermal conductivity $\kappa$, the Young modulus $E$ or the density of the bar $\rho$. The two simulations are illustrated in Figures 8 and 9 obtained for a bar with the same dimensions. The difference between the values of the first eigenfrequencies obtained with the two simulations is only of $45 \mathrm{~Hz}$. We can then safely use a full structure with adapted material parameters for the following simulations.

\section{B. Electromagnetic calculations}

A general electromagnetic calculation implies to solve the Maxwell equations

$$
\begin{gathered}
\vec{\nabla} \wedge \vec{H}=\vec{j}+\frac{\partial \vec{D}}{\partial t} \\
\vec{\nabla} \wedge \vec{E}=-\frac{\partial B}{\partial t}
\end{gathered}
$$

for the geometry given by one quarter of the bar + supporting arms. However, for our problem, additional assumptions

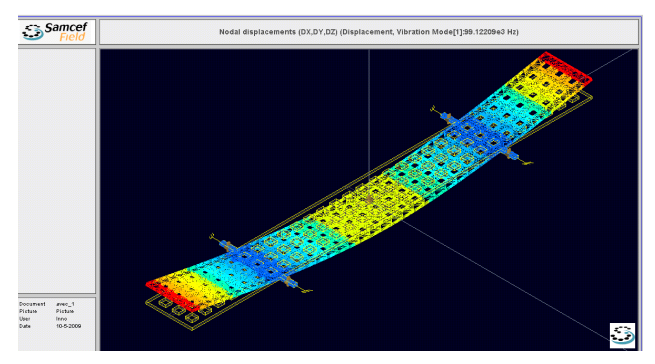

Fig. 8. Calculation of the first eigenfrequency of a structure with $2 \mu \mathrm{m} \times 2 \mu \mathrm{m}$ holes separated by a distance of $5 \mu \mathrm{m}$. Dimensions of the bar are $427 \mu \mathrm{m} \times 54 \mu \mathrm{m} \times 2.5 \mu \mathrm{m}$ and those of the supporting arms are $5 \mu \mathrm{m} \times 4 \mu \mathrm{m} \times 2.5 \mu \mathrm{m}$. The first eigenfrequency is $f=99.122 \mathrm{MHz}$.

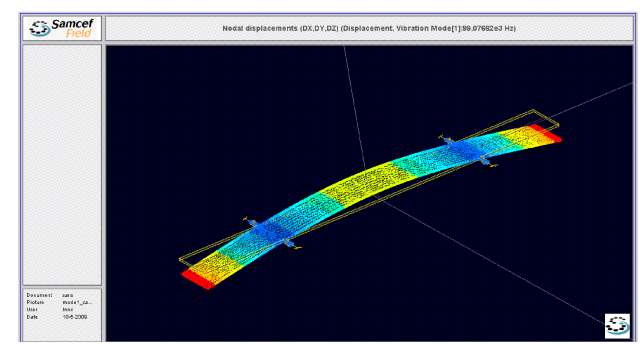

Fig. 9. Calculation of the first eigenfrequency of a structure with no holes and the same dimensions as in Figure 8. The properties of the material have been modified according to (12). The first eigenfrequency is $f=99.077$ MHz.

apply which simplify the Maxwell's equations. First, capacitive effects can be neglected, i.e. the displacement current $\frac{\partial \vec{D}}{\partial t}$ is negligible. This assumption holds because the size of the bar $L \ll \lambda$, the wavelength which is of the order of $3000 \mathrm{~m}$ for a vibration frequency of $\sim 100 \mathrm{KHz}$. Second, skin effects are negligible. Indeed the skin depth of the aluminium bar with the above dimensions is of the order of $300 \mu \mathrm{m}$, much larger than the thickness of the bar. In this case, the general electromagnetic problem reduces to an electrokinetic one and the Maxwell's equations become

$$
\begin{gathered}
\vec{\nabla} \cdot \vec{j}=0 \\
\vec{\nabla} \wedge \vec{E}=0
\end{gathered}
$$

From (15), the distribution of the current density throughout the bar can be calculated with adequate boundary conditions. Parallel electromagnetic boundary counditions were applied on external faces of the structure to simulate a null electromagnetic field at infinity. An example is shown in Figure 10. The distribution of the voltage along the structure can be obtained from (16). Finally the distribution of the magnetic field around the structure induced by the current flowing in the bar can also be calculated.

\section{Thermal calculations}

From the distribution of the current density $\vec{j}$, a thermal calculation can be performed assuming that heat is transmitted only by conduction. Neglecting thermal radiation is a valid 


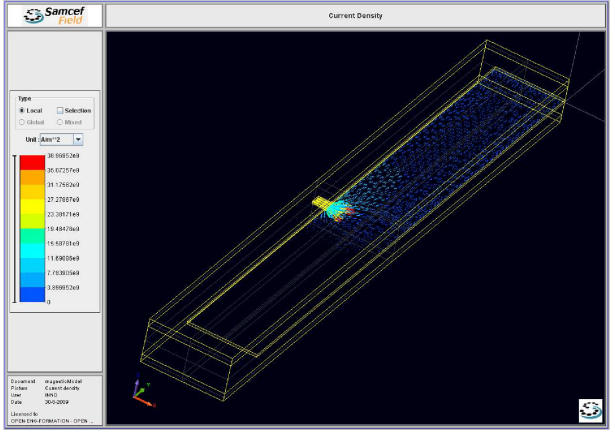

Fig. 10. Distribution of the current density in the bar obtained with Oofelie by solving an electrokinetic problem. Only one quarter of the bar is shown.

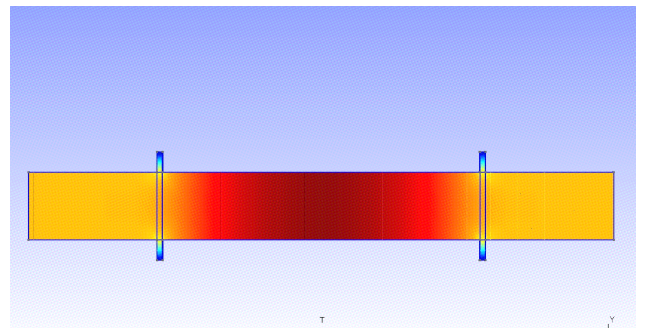

Fig. 11. Distribution of the temperature in a bar with dimensions $427 \mu \mathrm{m} \times$ $49 \mu \mathrm{m} \times 2.5 \mu \mathrm{m}$ and supporting arms of dimensions $15 \mu \mathrm{m} \times 4 \mu \mathrm{m} \times 2.5 \mu \mathrm{m}$. Applied voltage between the nodes is $\Delta V=0.0181 \mathrm{~V}$.

assumption given the size of the bar. Since we are not interested in the complete dynamical process but instead in the final thermo-electric steady-state equilibrium, only the steadystate equation of heat conduction is solved :

$$
\vec{\nabla} \cdot(\kappa \vec{\nabla} T)=-Q_{v}
$$

$Q_{v}$ is the power per unit volume generated as heat by the Joule effect. As mentionned above, in those simulations, the coupling between electric and thermal fields is neglected. An example of result is shown in Figure 11 for a bar with dimensions $427 \mu \mathrm{m} \times 49 \mu \mathrm{m} \times 2.5 \mu \mathrm{m}$ and supporting arms of dimensions $15 \mu \mathrm{m} \times 4 \mu \mathrm{m} \times 2.5 \mu \mathrm{m}$. The applied voltage between the nodes was $\Delta V=0.0181 \mathrm{~V}$, corresponding to a current of $\sim 0.15 \mathrm{~A}$. The maximum value of the temperature in the structure obtained with simulations is $296 \mathrm{~K}$, which is smaller than the value $(301.5 \mathrm{~K})$ obtained with the equivalent electric circuit method described in section II. The difference might be due to the lack of thermo-electrical coupling in our simulations but also to the too crude discretisation of the bar in the equivalent electric circuit. The discrepancy between results from the two methods increases when the applied voltage becomes larger. Figure 12 shows a profile in the temperature distribution in the bar taken at half the width of the bar.

\section{Mechanical calculations}

Firstly, the first eigenfrequency and the related mode shape are evaluated using the piezoelectric module of Oofelie. The external load applied to the bar is the Lorentz force, $\vec{F}=$ $\vec{j} \wedge \overrightarrow{B_{\text {ext }}}$, whose distribution along the bar is calculated from the

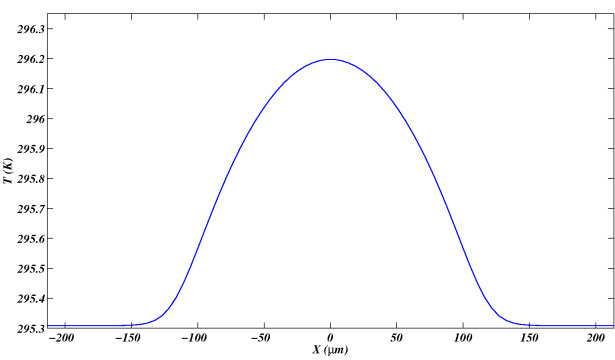

Fig. 12. Temperature profile along the bar corresponding to results shown in Figure 11. The cross section is located at half the width of the bar.

distribution of the current density $\vec{j}$ and from a given value of the external magnetic field $\overrightarrow{B_{\text {ext }}}$. The following finite elements equation is solved :

$$
M \ddot{\vec{q}}+C \dot{\vec{q}}+K \vec{q}=\vec{F}
$$

$M, C$, and $K$ respectively represent the mass matrix, the damping matrix and the spring matrix. $\vec{F}$ and $\vec{q}$ are respectively the vectors of applied forces and of the displacements of nodes/elements. For the damping matrix $C$, a proportional damping was assumed, i.e. $C=a_{1} M+a_{2} K$, from which we obtain $\frac{1}{2 Q} \simeq \epsilon=\frac{1}{2 \omega} a_{1}+\frac{1}{2} a_{2} \omega$, where $\epsilon$ is the damping ratio. $Q$ can be found either with analytical calculations (as has been done in section III) or by evaluating it with dynamic simulations (see determination of the thermoeleastic damping below). From $Q$, the values of $a_{1}$ and $a_{2}$ to use in the simulations can be determined.

The size of the mesh must be checked in order to see if the discretisation of the system is good enough. For that purpose, the first eigenfrequency is calculated with an increasing number of elements until it converges. The discretisation is sufficient when convergence is reached. Tetrahedral elements are used. Equation (18) is solved with a Cholesky LU factorization solver. Convergence was better achieved with second order elements. Typical number of elements needed to reach convergence is of the order of $10^{5}$.

Then, a frequency sweep around the first eigenfrequency is carried out to study the dynamic behavior of the bar. An example is shown in Figure 13 where the deflection of the bar is shown as a function of the frequency for a given value of $Q=10000$. The simulations results are in better agreement with the theoretical curve given by equation (3).

On the other hand, the theoretical value of the first eigenfrequency, given by equation (1), is very different from the value obtained with Oofelie simulations. This is highlighted in table I where theoretical and simulated values of the first eigenfrequency have been calculated for various lengths of the bar. The discrepancy between the theoretical and simulated values are probably due to the fact that the true geometry of the bar is more complex than a free-free bar.

Table II compares theoretical and simulated values of the first eigenfrequencies of the bar for several values of the bar width. The theoretical value given by (1) is independent of the width of the bar. However, simulations show that the width of 


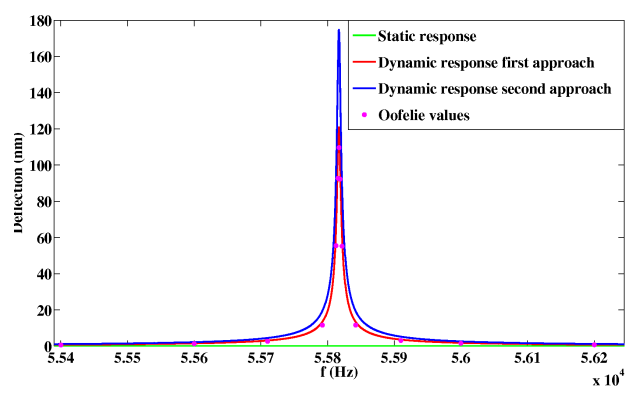

Fig. 13. Deflection of the bar as a function of the frequency for a given value of $Q=10000$. Dimensions of the aluminium bar are $411 \mu \mathrm{m} \times 54 \mu \mathrm{m} \times 1 \mu \mathrm{m}$ and those of the supporting arms are $5 \mu \mathrm{m} \times 4 \mu \mathrm{m} \times 1 \mu \mathrm{m}$. The corresponding first eigenfrequency is $55,817 \mathrm{KHz}$. Dots are values of simulations obtained with Oofelie. The red curve is the theoretical dynamical response given by equation (3) while the blue curve is the theoretical dynamical response given by equation (4).

\begin{tabular}{ccc}
\hline $\begin{array}{c}\text { Length } \\
\mu \mathrm{m}\end{array}$ & $\begin{array}{c}\text { Theorerical value } \\
\mathrm{KHz}\end{array}$ & $\begin{array}{c}\text { Simulation value } \\
\mathrm{KHz}\end{array}$ \\
\hline & & \\
215 & 283.405 & 343.380 \\
313 & 133.709 & 171.452 \\
411 & 77.553 & 104.439 \\
509 & 50.564 & 71.165 \\
607 & 35.555 & 52.130 \\
705 & 26.357 & 40.183 \\
803 & 20.316 & 32.179 \\
901 & 16.135 & 26.549 \\
\hline
\end{tabular}

TABLE I

THEORETICAL AND NUMERICAL VALUES OF THE FIRST EIGENFREQUENCIES AS A FUNCTION OF THE LENGTH OF AN ALUMINIUM BAR WITH WIDTH $a=54 \mu \mathrm{m}$, THICKNESS $b=2.5 \mu \mathrm{m}$, AND WITH $15 \mu \mathrm{m} \times 4 \mu \mathrm{m} \times 2.5 \mu \mathrm{m}$ SUPPORTING ARMS.

\begin{tabular}{ccc}
\hline $\begin{array}{c}\text { Width } \\
\mu \mathrm{m}\end{array}$ & $\begin{array}{c}\text { Theorerical value } \\
\mathrm{KHz}\end{array}$ & $\begin{array}{c}\text { Simulation value } \\
\mathrm{KHz}\end{array}$ \\
\hline & & \\
26 & 51.984 & 88.462 \\
54 & 51.984 & 70.323 \\
103 & 51.984 & 64.374 \\
152 & 51.984 & 61.228 \\
201 & 51.984 & 59.400 \\
\hline
\end{tabular}

TABLE II

THEORETICAL AND NUMERICAL VALUES OF THE FIRST EIGENFREQUENCIES AS A FUNCTION OF THE WIDTH OF AN ALUMINIUM BAR WITH LENGTH $L=502 \mu \mathrm{m}$, THICKNESS $b=2.5 \mu \mathrm{m}$, AND WITH $15 \mu \mathrm{m} \times 4 \mu \mathrm{m} \times 2.5 \mu \mathrm{m}$ SUPPORTING ARMS.

the bar is an important parameter for the determination of the eigenfrequencies, supporting the idea that our system cannot be modelled by a simple free-free bar.

Finally, let us note that the sinusoidal current in the bar induces a magnetic field around it which in magnitude may be much larger than the external magnetic field we wish to measure. However, this induced magnetic field also varies sinusoidally and the resulting Lorentz force varies therefore as $\sim \sin ^{2} \omega t \propto \frac{1+\cos 2 \omega t}{2}$. As a consequence, this force is not amplified since it has a static component and a component vibrating at twice the first eigenfrequency of the structure. Moreover, the distribution of the induced magnetic field is symmetric if the shape of the bar is symmetric. During small displacements, this symmetry is slightly broken but the effect is very small. On the other hand, if there are larger asymmetries in the bar due to the micromachining process, the influence of the induced magnetic field can set up an upper limit on the smallest value of the external magnetic field that can be measured with this technique.

\section{E. Multi-layer structures}

A prototype bar with multi-layer structure has also been manufactured at the Microwave Laboratory of UCL (see Section V). Therefore simulations with multi-layer structures (2 or 3) were also carried out with Oofelie, one layer being made of aluminium and the other ones made of less conductive material such as silicon or silicon nitride $\left(\mathrm{Si}_{3} \mathrm{~N}_{4}\right)$.

1) Electromagnetic and thermal calculations: The electromagnetic and thermal simulations can be done similarly as for monolayer structures. The current density $\vec{j}$ is mainly distributed within the most conductive material (aluminium). Thermal fluxes are also predominant in the aluminium layer.

2) Mechanical calculations: Eigenfrequencies increase in multi-layer structures in comparison to the values obtained for mono-layer structures. For example, for a $411 \mu \mathrm{m} \times 54 \mu \mathrm{m} \times$ $1 \mu \mathrm{m}$ with $5 \mu \mathrm{m} \times 4 \mu \mathrm{m} \times 1 \mu \mathrm{m}$ supporting arms, the first eigenfrequency is $55.818 \mathrm{KHz}$ for a mono-layer structure, 89.358 $\mathrm{KHz}$ for a two-layer aluminium/silicon nitride structure and $92.609 \mathrm{KHz}$ for a three-layer aluminium/silicon nitride/silicon structure.

With multi-layer structures, residual stresses can occur due to intrinsic or extrinsic phenomena. To establish the accurate distribution of these stresses is a very complicated task which can be modeled with FEM simulations only if exact details of the manufacturing processes are known. As a first step, a simpler approach is undertaken : only residual stresses due to a mismatch between aluminium and silicon nitride thermal coefficients is considered. Figure 14 illustrates the deformation of a bi-layer structure due to cooling from $150^{\circ} \mathrm{C}$, the deposition temperature for aluminium, to an ambient temperature of $20^{\circ} \mathrm{C}$. The nitride layer undergoes compressive stresses while the aluminium one undergoes tensile stresses. The first eigenfrequency is shifted from $\sim 90 \mathrm{KHz}$ for the structure without these residual stresses to $\sim 105 \mathrm{KHz}$ when these residual stresses are taken into account. Further simulations including detailed residual stresses in the bar will be important to accurately model the dynamic behavior of the structure.

Finally, even though TED is negligible in mono-layer structure with thicknesses lower or equal to $2.5 \mu \mathrm{m}$, it has to be checked for bi-layer and three-layer structures. We assume other types of dampings are negligible. The pyropiezoelectric module of Oofelie is used as it allows couplings between thermal and mechanical fields and therefore the prediction of the TED. A frequency sweep around the first eigenfrequency with $\Delta f=7 \mathrm{~Hz}$ gives the deformation of the bar as a function 


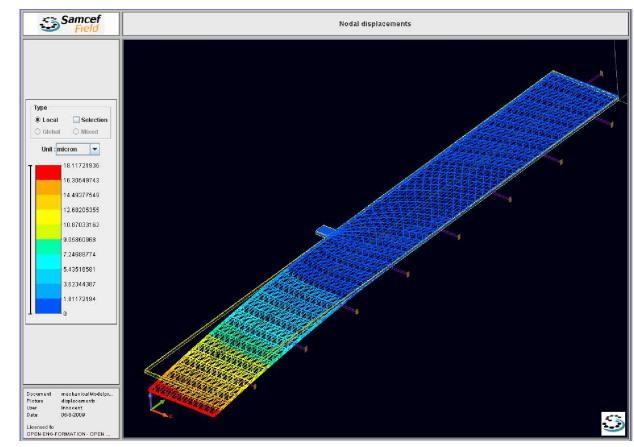

Fig. 14. Deformation of a $411 \mu \mathrm{m} \times 54 \mu \mathrm{m} \times 1.25 \mu \mathrm{m}$ bar with $5 \mu \mathrm{m} \times$ $4 \mu \mathrm{m} \times 1.25 \mu \mathrm{m}$ supporting arms. The structure has two layers : $1 \mu \mathrm{m}$ layer of aluminium and $0.25 \mu \mathrm{m}$ layer of silicon nitride.

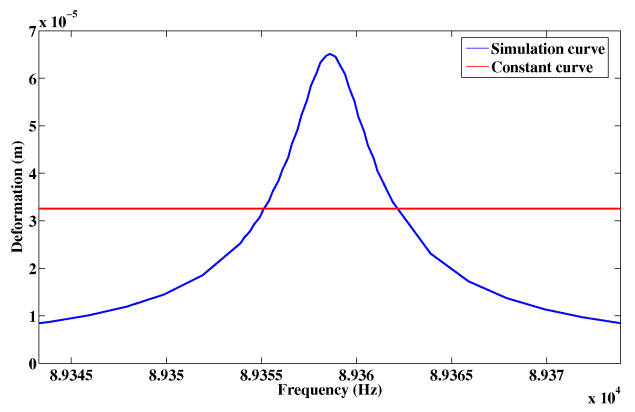

Fig. 15. Deformation of a $411 \mu \mathrm{m} \times 54 \mu \mathrm{m} \times 1.25 \mu \mathrm{m}$ two-layer bar $(1 \mu \mathrm{m}$ layer of aluminium and $0.25 \mu \mathrm{m}$ layer of silicon nitride) with $5 \mu \mathrm{m} \times 4 \mu \mathrm{m} \times$ $1.25 \mu \mathrm{m}$ supporting arms, as a function of the frequency. $\Delta f=7 \mathrm{~Hz}$.

of the frequency. Results for a $411 \mu \mathrm{m} \times 54 \mu \mathrm{m} \times 1.25 \mu \mathrm{m}$ twolayer bar ( $1 \mu \mathrm{m}$ layer of aluminium and $0.25 \mu \mathrm{m}$ of nitride) with $5 \mu \mathrm{m} \times 4 \mu \mathrm{m} \times 1.25 \mu \mathrm{m}$ supporting arms are shown in Figure 15. The value of the quality factor $Q$ is obtained from

$$
Q=\frac{\omega_{0}}{\Delta \omega}
$$

where $\omega_{0}$ is the frequency of the peak deformation $d_{\max }$ and $\Delta \omega$ is the frequency width at a value of $\frac{d_{\max }}{\sqrt{2}}$. A value of $Q=12765$ is found. For comparison, in mono-layer structures, a value of $Q \sim 558000$ is found for a structure with the same dimensions (except the thickness $b$ was equal to $1 \mu \mathrm{m}$ and made only of aluminium) and with the same assumptions (no other type of dampings). For a three-layer structure with the same dimensions and a thickness of $1.35 \mu \mathrm{m}(1 \mu \mathrm{m}$ of aluminium, $0.25 \mu \mathrm{m}$ of silicon nitride and $0.1 \mu \mathrm{m}$ of silicon), the value of the quality factor is $Q=13153$. Thermoelastic damping is therefore an important source of damping in these multi-layer structures.

\section{FABRICATION OF A XBM PROTOTYPE}

Following the parametric study and the simulations of the XBM, prototypes have been realized in the Microwave Laboratory of UCL. Two fabrication processes have been used : Silicon-On-Insulator (SOI) and bulk. Several materials were used, including aluminium, silicon and insulators such as silicon nitride $\left(\mathrm{Si}_{3} \mathrm{~N}_{4}\right)$ and silicon oxide $\left(\mathrm{SiO}_{2}\right)$. With both

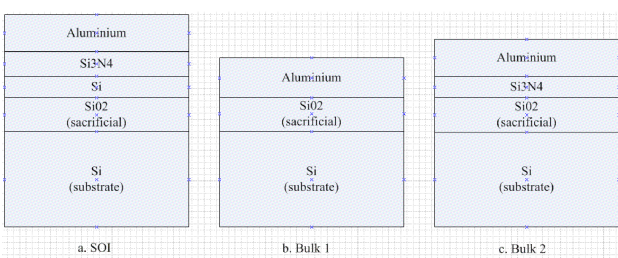

Fig. 16. Design of the different layers used in the various fabrication processes for the XBM prototype

processes, the curvature of the structure must be as small as possible. Curvature of the structure may arise due to stress gradients developing along the thickness of mono- or multilayer structures. The origin of these stress gradients can be either intrinsic or extrinsic. In the latter case, they are due to a difference in the thermal expansion coefficients between the various layers constituting the structure. Indeed, these layers are deposited at high temperature and contract differently when they cool down. This creates stresses because the layers stick together.

Figure 16 illustrates the three classes of structures designed for the XBM prototype. For the SOI structures, we start with a material made of $100 \mathrm{~nm}$ silicon layer - $250 \mathrm{~nm} \mathrm{SiO} 2$ layer $380 \mu \mathrm{m}$ silicon (substrate) on top of which is deposited a 250 $\mathrm{nm}$ LPCVD (low pressure chemical vapor deposition) silicon nitride film. A mask is used to etch both the nitride and silicon layers using positive litography. An $880 \mathrm{~nm}$ thick aluminium layer is then deposited on top of the structure with an electron gun deposition technique. The oxide layer is finally removed using HF.

For bulk processes, there are two different types of structures. In both types, a thick bulk silicon layer is the substrate on top of which a $400 \mathrm{~nm}$ silicon oxide film is deposited. In the first type of structure, a $1000 \mathrm{~nm}$ aluminium film is deposited using the electron gun deposition technique, then the $\mathrm{SiO}_{2}$ film is etched. In the second type of structures, a $250 \mathrm{~nm}$ LPCVD nitride film is deposited between the silicon oxyde film and the aluminium layer. It is etched before the oxide film.

With bulk micromachining processes, the use of aluminium which has much larger electric and thermal conductivites than silicon increases the maximum value of current which can flow through the bar, and therefore increase the sensitivity of the device. However, an important drawback is that large residual stresses remain after the fabrication process compared to those observed in SOI structures. These stresses are mainly due to the large difference of thermal expansion coefficients between the silicon substrate and the aluminium layer.

In addition to the three different structures described above, several lengths and widths of the bar are considered. Square holes of dimensions $2 \mu \mathrm{m} \times 2 \mu \mathrm{m}$ and spaced by $5 \mu \mathrm{m}$ have been included in the design to allow the etching of the insulating layer underneath the structure to release it.

These XBM prototypes will be tested soon at the LTAS and results will be compared to the analytical results and the simu- 


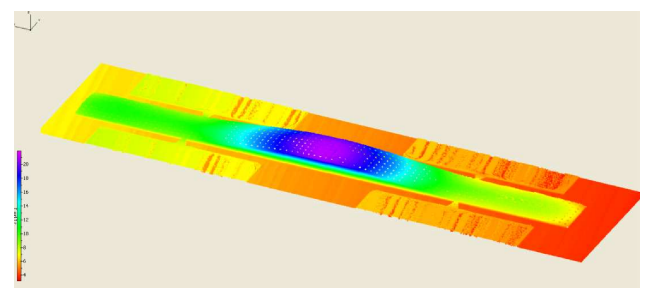

Fig. 17. Topography of one of the MEMS XBM prototype obtained with white-light interferometry technique.

lations obtained with Oofelie. Measurements of the dimensions of the curvature of the structure have been conducted at UCL with the Polytec MSA-500 machine which uses white-light interferometry techniques to measure the topography of the structure. An example is shown in Figure 17. The structure is strongly curved in the central part, with an elevation of a few $\mu \mathrm{m}$. Future measurements will have to determine how the curvature of this structure affects the determination of the eigenfrequencies of the bar as well as its displacement.

\section{Methods FOR DETECTING THE BAR DEFLECTION}

According to equation (3), if we know the dimensions of the bar, the Young modulus of the material, the electric current flowing through the device and the mechanical quality factor at the resonance, one component of the external magnetic field can be determined from the measurement of the bar displacement $d$. In this section, we review a number of wellestablished detection techniques which can be used to measure this displacement such as optical and capacitive methods. We also briefly consider potentially interesting new techniques such as surface plasmons resonance and the use of a magnetostrictive material for the bar. An important aspect is that we are looking for a device that can be miniaturized together with the bar and the associated electronics.

\section{A. Optical methods}

The first optical method proposed here consists in measuring the time delay needed by a laser beam emitted by a laser diode to return after a reflection on one point of the bar. This method will be used in the LTAS to measure the bar displacement of the XBM prototypes. A variant of this method was used by the team at the John Hopkins Institute (Oursler et al, 1999). In this case, the light from the laser diode is reflected from the bar to form a spot on a position-sensitive-device. In general, optical methods can reach a resolution lower than $1 \mathrm{~nm}$ but the optical devices cannot easily be minituarized.

\section{B. Capacitive methods}

With this method, the variation of a capacitance $C$ is measured to determine the displacement of the bar. The bar is used as one of the capacitor's electrode and the other (static) electrode is placed under the bar on the silicon substrate. First, the value of the capacitance is measured when the bar is static. Then, when the bar undergoes deformation, the upper electrode moves and there is a change of the capacitance $\Delta C$ linked to the displacement of the bar. For a parallel-plate capacitor, when the surface of the electrodes $S_{e}$ is much larger than the distance between them $d\left(S_{e} \gg d^{2}\right)$, the value of the capacitance is simply given by

$$
C=\frac{\epsilon S_{e}}{d}
$$

where $\epsilon$ is the dielectric permittivity of the medium between the electrodes.

There are a number of difficults to solve associated to this method. First, the variation of $C$ with $d$ is not linear but hyperbolic. A way to circumvent this problem is to actually measure the impedance $X=1 / \omega C$ rather than the capacitance itself. An example is to use a Wheatstone bridge in a "push-pull" configuration in which two capacitors are located in contiguous branches and similar impedances are chosen for the two other branches. The bridge is in equilibrium in the static case. When the bar vibrates and moves of a distance $d$, the value of the first capacitance becomes $C_{1}=\frac{\epsilon S_{e}}{d_{s t}+d}$ while the value of the other one becomes $C_{2}=\frac{\epsilon S_{e}}{d_{s t}-d}$ where $d_{s t}$ corresponds to the static case. The new equilibrium condition gives an output voltage at the detector which is directly proportional to the bar displacement (if $d \ll d_{s t}$, the distance between the electrodes in the static case). Second, when the bar vibrates, the form of the capacitor is different from a perfect parallel-plate capacitor. The distance $d$ between the bar and the substrate is not the same along the bar since it has a parabolic shape. This makes it more difficult to actually calculate the value of the capacitance. Also, the assumption $S \gg d^{2}$ has to be carefully checked in order to see if fringing effects are indeed negligible, otherwise equation (20) is not valid anymore. Finally, to accurately measure the small value of $\Delta C$ resulting from the tiny displacement of the bar $d$, the shielding of the wires must be really good to minimize parasitic capacitances.

Capacitance variations are usually converted into a voltage or a frequency with electric circuits. With capacitive methods, subnanometric distances can be measured. For a $50 \mu \mathrm{m}$ wide beam and an initial gap of $1 \mu \mathrm{m}$ between the bar and the substrate (or between the electrodes), the initial (static) capacitance is $C \sim 2 \times 10^{-2} \mathrm{pF}$. The variation of the capacitance $\Delta C$ due to a $10 \mathrm{~nm}$ displacement of the bar is of the order of $2 \times 10^{-4} \mathrm{pF}$. Despite some difficulties, one of the strong advantage of capacitive methods is their capabilities of miniaturization.

\section{Use of a magnetostrictive material}

If the bar is made of a magnetostrictive material, its dimensions and properties will change in the presence of an external magnetic field which will modify eigenfrequencies according to equation (1). With this technique, a frequency shift is measured instead of the displacement of the bar which is easier to measure accurately. Magnetostrictive materials are characterized by their magnetostrictive coefficient $\Lambda$ which measures the fractional change in length when the magnetization of the material increases from 0 to its saturation value. This coefficient is usually of the order of $10^{-5}$ such that the 
frequency shifts due to subnanotesla values would be very small. Magnetostrictive alloys with higher magnetostrictive coefficients do exist but are not widely used in MEMS technology.

\section{Surface Plasmons Resonance}

Surface plasmons (SP) are collective surface plasma oscillations of the free electron gas on the interface between a metal and a dielectric medium. Those electromagnetic waves propagate parallel to the metal/dielectric boundary and are very sensitive to any change of this boundary. SP can be excited resonantly by TM (transverse magnetic) polarized light hitting the interface at a specific incident angle when the tangential component of its wave vector matches the SP wave vector $k_{\mathrm{SP}}$. When this happens, the light intensity reflected by the metallic film is strongly dimmed for this specific angle (Hastanin et al, 2008). The wave vector of the SP is given by the following dispersion relation :

$$
k_{\mathrm{SP}}=\frac{\omega}{c} \sqrt{\frac{\epsilon(\omega) \cdot \epsilon_{d}}{\epsilon(\omega)+\epsilon_{d}}}
$$

where $\omega / c$ is the wave vector of the light in the vacuum, $\epsilon(\omega)$ is the complex dielectric function of the metal and $\epsilon_{d}$ is the dielectric function of the dielectric medium. The wave vector of SP is larger than the tangential component of the wave number of the incident light for any given incident angle. Therefore, the SP can be excited only by an evanescent light wave. The most common way to excite SP is the KretschmannRaether configuration in which TM polarized light coming from a source is totally reflected on the surface of a prism onto which a thin metal film has been deposited (Kretschmann \& Raether 1968). The evanescent wave interacts with the plasma waves on the surface and generates plasmons. In this case, the surface plasmon resonance (SPR) incident angle is given by

$$
\theta_{\mathrm{SPR}}=\arcsin \left(\frac{1}{n} \sqrt{\frac{\epsilon_{r}(\omega) \cdot \epsilon_{d}}{\epsilon_{r}(\omega)+\epsilon_{d}}}\right)
$$

where $n$ is the refraction index of the prism and $\epsilon_{r}(\omega)$ is the real part of the dielectric function of the metal.

Both the wave vector of SP and the SPR angle of incidence depends on the thickness of the dielectric medium. Now let us locate the vibrating bar in the vicinity of this device at a distance $d_{1}$ of the thin metal film. In the static case, the SPR incident angle as well as the sharp dip of the intensity of the light can be determined. In the dynamical case, when the bar vibrates, the thickness $d_{1}$ of the dielectric medium (air or vacuum) will change accordingly to the deformation of the bar $d$ and produce a SPR angle shift sufficient to be detected via the metal film reflectivity measurement (Hastanin et al, 2008).

Typical metals that support surface plasmons are silver, gold or copper. Gold and copper are used for MEMS manufacturing. Both metals have a better electrical conductivity than aluminium. Copper is also a slightly stronger material than pure aluminium but does not adhere particularly well to silicon. Copper is also an excellent thermal conductor. SPR is a promising method for detecting the bar displacement but since the readout detection measurement relies on optical methods, it might prove difficult to be miniaturized.

\section{CONCLUSIONS AND PERSPECTIVES}

In space physics community, there is an increasing need to carry out multi-point measurements, in particular for magnetic field measurements. This is true for both scientific and space weather applications. The current trend is to develop and launch a set of micro-, nano- or pico-satellites carrying a small and light payload with small energy consumptions needs. With these requirements in mind, we initiated a study to design a MEMS xylophone bar magnetometer which fulfills these conditions.

Of course the XBM must also be able to at least reach the same accuracy as magnetometers currently flying in space, i.e. a precision of $\sim 0.1 \mathrm{nT}$. With typical dimensions used in this paper for an aluminium bar ( $L \sim 500 \mu \mathrm{m}, a \sim 50 \mu \mathrm{m}$ and $b \sim 1 \mu \mathrm{m}$ ) and with a maximal RMS value of $0.15 \mathrm{~A}$ for the current flowing through the bar, the displacement of the bar is very small, $d \sim 10^{-11} \mathrm{~m}$, in the presence of an external magnetic field $B_{\text {ext }}=0.1 \mathrm{nT}$ and for a reasonable quality factor $Q=10000$. Measuring such a small value of the bar displacement will be a very challenging task.

In the near future, FEM simulations will be improved to better take into account the complex couplings between the electromagnetic, thermal and mechanical fields. A detailed modelisation of the manufacturing process used to build the MEMS XBM will also be carried out with FEM in order to obtain an accurate distribution of residual stresses in the bar and see how this distribution modifies its eigenfrequencies. Those simulations will be compared to measurements obtained at LTAS on the XBM prototype manufactured at the Microwave Laboratory of UCL.

\section{ACKNOWLEDGMENT}

The authors would like to thank the company "Open Engineering" for the use of the Oofelie software and in particular Stéphane Paquay for his numerous advices on how to carry out and improve the FEM simulations. The authors would also like to thank the group of "Electrical engineering and Computer Science", ACE, of Professor Geuzaine for their precious help in electromagnetic simulations. Finally, the authors would like to thank Professor Raskin and his team from the Microwave Laboratory of UCL for the access to their facilities and the manufacturing of our XBM prototypes. Véronique Rochus acknowledges the financial support of the Belgian National Fund for Scientific Research.

\section{REFERENCES}

[1] Blom F.R. et al, Dependence of the quality factor of micromachined silicon beam resonators on pressure and geometry, J. Vac. Sci. Technol., Vol B10, 19-26, 1992.

[2] Givens R.B. et al, A high sensitivity, wide dynamic range magnetometer designed on a xylophone resonator, Appl. Phys. Lett. 69 (18), 27552757, 1996. 
[3] Hastanin J. et al, A gas micromechanical sensor based on surface plasmons resonance, Sensors \& Transducers Journal, Vol. 96, Issue 9, 8-17, 2008.

[4] Hosaka H. et al, Damping characteristics of beam-shaped microoscillators, Sens. Actuators, vol. A49, 87-95, 1995.

[5] Kretschmann E. \& Raether H., Radiative decay of non-radiative surface plasmons excited by light, Z. Naturforsch, 23A, 2135-2136, 1968.

[6] Lifshitz R. \& Roukes M.L., Thermoelastic damping in micro- and nanomechanical systems, Physical Review B, Volume 61, number 8, $5600-5609,2000$.

[7] Naeli K. \& Brand O., Dimensional considerations in achieving large quality factors for resonant silicon cantilevers in air, Journal of Applied Physics, 105, 014908, 2009.

[8] Niyonzima I., Design and simulation of a MEMS magnetometer : the Xylophone Bar Magnetometer, master thesis in order to obtain a Master Degree in Engineering Physics at the University of Liège, 2009

[9] D.A. Oursler et al, Development of the Johns Hopkins Xylophone Bar Magnetometer, John Hopkins APL Technical Digest, Volume 20, Numero 2, 1999.

[10] Wickenden D.K. et al, Development of miniature magnetometers, John Hopkins APL Technical Digest, Volume 18, Number 2, 1997.

[11] Wickenden D.K. et al, Micromachined polysilicon resonating xylophone bar magnetometer, Acta Astronautica 52, 421-425, 2003.

[12] Yang J. et al, Energy dissipation in submicrometer thick single-crystal silicon cantilevers, Journal of Microelectromechanical systems, Vol 11, 775-783, 2002.

[13] Zanetti L.J. et al, Miniature magnetic field sensors based on xylophone resonators, Science closure and Enabling technologies for Constellation Class Missions, edited by V. Angelopoulos and P.V. Panetta, 149-151, UC Berkeley, Californie, 1998. 